\title{
System of nonlinear variational inclusion problems with $(A, \eta)$-maximal monotonicity in Banach spaces
}

\author{
N. K. Sahu ${ }^{1, *}$, N. K. Mahato ${ }^{2}$, Ram N. Mohapatra ${ }^{3}$ \\ ${ }^{1}$ Dhirubhai Ambani Institute of Information and Communication Technology, Gandhinagar, India \\ 2 PDPM IIITDM, Jabalpur, India \\ ${ }^{3}$ University of Central Florida, Orlando, FL. 32816, USA
}

(Received: 29 June 2016; Accepted: 20 June 2017)

\begin{abstract}
This paper deals with a new system of nonlinear variational inclusion problems involving $(A, \eta)$-maximal relaxed monotone and relative $(A, \eta)$-maximal monotone mappings in 2-uniformly smooth Banach spaces. Using the generalized resolvent operator technique, the approximation solvability of the proposed problem is investigated. An iterative algorithm is constructed to approximate the solution of the problem. Convergence analysis of the proposed algorithm is investigated. Similar results are also proved for other system of variational inclusion problems involving relative $(A, \eta)$ maximal monotone mappings and $(H, \eta)$-maximal monotone mappings.
\end{abstract}

Keywords Variational inclusion, Generalized resolvent operator, 2-uniformly smooth Banach space, Semi-inner product space

AMS 2010 subject classifications 47J22, 47J20, 46C50

DOI: $10.19139 /$ soic.v5i3.238

\section{Introduction}

Variational inequalities have been well studied and generalized to different directions due to its large association with partial differential equations and optimization problems. Variational inclusion problem is a natural generalization of variational inequality problem and it is of recent interest. Monotonicity plays a prominent role in the solvability of variational inclusion problem. Researchers have been studied different kind of monotonicity such as maximal monotonicity, relaxed monotonicity, $H$-monotonicity, $A$-monotonicity etc., and solved different variational inclusion problems taking the help of such monotonicity of the underlying operator. The method based on the resolvent operator technique is a generalization of projection method and has been widely used to solve variational inclusion problems (see [1], [3][5], [6], [7], [9], [11] and [12]).

Peng and Zhu [14] introduced and studied a new system of variational inclusion problems involving $(H, \eta)$ monotone operators. Verma [16] solved a new class of set valued variational inclusions involving $(A, \eta)$-monotone operators in Hilbert space. He also studied the notion of $(A, \eta)$-maximal relaxed monotonicity in Verma [17]. He examined the sensitivity analysis for quasi-variational inclusion problems using $(A, \eta)$-maximal relaxed monotonicity. Recently, Agarwal and Verma [2] solved a new system of variational inclusion problems involving $(A, \eta)$-maximal relaxed monotone mappings and relative $(A, \eta)$-maximal monotone mappings in Hilbert space.

\footnotetext{
${ }^{*}$ Correspondence to: N. K. Sahu (Email: nabindaiict@gmail.com). Dhirubhai Ambani Institute of Information and Communication
} Technology, Gandhinagar, India.

ISSN 2310-5070 (online) ISSN 2311-004X (print)

Copyright (C) 2017 International Academic Press 
More recently, Sahu et al. [15] have studied a class of $A$-monotone implicit variational inclusion problems in semi-inner product spaces.

In this paper, we generalize the work of Agarwal and Verma [2] from Hilbert space to 2-uniformly smooth Banach space. Although the results are similar, here we use different notion and techniques to solve those system of variational inclusion problems in 2-uniformly smooth Banach space. People generally take the help of bounded linear functionals to study those type of problems in Banach space. Instead of using arbitrary bounded linear functionals, we take the help of semi-inner product structure introduced by Lumer [13]. Semi-inner product helps us in a number of aspects for the smooth study of the problem. So first we afford a view of semi-inner product and its important features that we use in our work.

\section{Definition 1.1}

(Lumer [13]) Let $\mathrm{X}$ be a vector space over the field $\mathrm{F}$ of real or complex numbers. A functional [., .] $: X \times X \rightarrow F$ is called a semi-inner product if it satisfies the following:

1. $[x+y, z]=[x, z]+[y, z], \forall x, y, z \in X$;

2. $[\lambda x, y]=\lambda[x, y], \forall \lambda \in F$ and $x, y \in X$;

3. $[x, x]>0$, for $x \neq 0$;

4. $|[x, y]|^{2} \leqslant[x, x][y, y]$.

The pair $(X,[.,]$.$) is called a semi-inner product space.$

A semi-inner product space is a normed linear space with the norm $\|x\|=[x, x]^{\frac{1}{2}}$. Every normed linear space can be made into a semi-inner product space in infinitely many different ways. Giles [8] had shown that if the underlying space $X$ is a uniformly convex smooth Banach space then it is possible to define a semi-inner product uniquely. For a detailed study and fundamental results on semi-inner product spaces, one may refer to Lumer [13], Giles [8] and Koehler [10].

\section{Example 1.1}

The real Banach space $L^{p}(\mathbb{R})$ for $1<p<\infty$ is a uniformly convex smooth Banach space with a unique semi-inner product defined by

$$
[f, g]=\frac{1}{\|g\|_{p}^{p-2}} \int_{\mathbb{R}} f(t)|g(t)|^{p-1} \operatorname{sgn}(g(t)) d t, \quad f, g \in L^{p}(\mathbb{R}) .
$$

\section{Example 1.2}

The real sequence space $l^{p}$ for $1<p<\infty$ is a uniformly convex smooth space with a unique semi-inner product defined by

$$
[x, y]=\frac{1}{\|y\|_{p}^{p-2}} \sum_{i} x_{i} y_{i}\left|y_{i}\right|^{p-2}, x, y \in l^{p}
$$

\section{Definition 1.2}

(Xu [18]) Let $X$ be a real Banach space. The modulus of smoothness of $X$ is defined as

$$
\rho_{X}(t)=\sup \left\{\frac{\|x+y\|+\|x-y\|}{2}-1:\|x\|=1,\|y\|=t, t>0\right\} .
$$

$X$ is said to be uniformly smooth if $\lim _{t \rightarrow 0} \frac{\rho_{X}(t)}{t}=0$.

$X$ is said to be $p$-uniformly smooth if there exists a positive real constant $c$ such that $\rho_{X}(t) \leq c t^{p}, p>1$.

$X$ is said to be 2-uniformly smooth if there exists a positive real constant $c$ such that $\rho_{X}(t) \leq c t^{2}$.

Lemma 1.1

(Xu [18]) Let $X$ be a smooth Banach space. Then the following statements are equivalent:

(i) $X$ is 2-uniformly smooth.

(ii) There is a constant $c>0$ such that for every $x, y \in X$, the following inequality holds

$$
\|x+y\|^{2} \leq\|x\|^{2}+2\left\langle y, f_{x}\right\rangle+c\|y\|^{2},
$$


where $f_{x} \in J(x)$ and $J(x)=\left\{x^{*} \in X^{*}:\left\langle x, x^{*}\right\rangle=\|x\|^{2}\right.$ and $\left.\left\|x^{*}\right\|=\|x\|\right\}$ is the normalized duality mapping, where $X^{*}$ denotes the dual space of $X$ and $\left\langle x, x^{*}\right\rangle$ denotes the value of the functional $x^{*}$ at $x$, that is $x^{*}(x)$.

\section{Remark 1.1}

Every normed linear space is a semi-inner product space (see Lumer [13]). In fact by Hahn Banach theorem, for each $x \in X$, there exists at least one functional $f_{x} \in X^{*}$ such that $\left\langle x, f_{x}\right\rangle=\|x\|^{2}$. Given any such mapping $f$ from $X$ into $X^{*}$, it has been verified that $[y, x]=\left\langle y, f_{x}\right\rangle$ defines a semi-inner product. Hence we can write the inequality $(1.1)$ as

$$
\|x+y\|^{2} \leq\|x\|^{2}+2[y, x]+c\|y\|^{2}, \forall x, y \in X .
$$

The constant $c$ is chosen with best possible minimum value. We call $c$, as the constant of smoothness of $X$.

Example 1.3

The functions space $L^{p}$ is 2 -uniformly smooth for $p \geq 2$ and it is $p$-uniformly smooth for $1<p<2$.

If $2 \leq p<\infty$, then we have for all $x, y \in L^{p}$,

$$
\|x+y\|^{2} \leq\|x\|^{2}+2[y, x]+(p-1)\|y\|^{2} .
$$

Here the constant of smoothness is $p-1$.

\section{Preliminaries}

In the vicinity of the paper, we assume that $X$, is a real 2-uniformly smooth Banach space endowed with norm $\|$. and semi-inner product [., .], $d$ is the metric induced by the norm, $2^{X}$ is the power set of $X, C B(X)$ is the set of all closed and bounded subsets of $X$ and $D(.,$.$) is the Housdorff metric on C B(X)$ defined by

$$
D(A, B)=\max \left\{\sup _{x \in A} d(x, B), \sup _{y \in B} d(A, y)\right\}
$$

where $d(x, B)=\inf _{y \in B} d(x, y)$ and $d(A, y)=\inf _{x \in A} d(x, y)$.

\section{Definition 2.1}

Let $T, A: X \rightarrow X$ be single valued mappings. The operator $T$ is said to be

(i) monotone if $[T x-T y, x-y] \geq 0, \forall x, y \in X$;

(ii) monotone with respect to $A$ if $[T x-T y, A x-A y] \geq 0, \forall x, y \in X$;

(iii) strictly monotone if $T$ is monotone and $[T x-T y, x-y]=0$ if and only if $x=y$;

(iv) $r$-strongly monotone if there exists a constant $r>0$ such that

$$
[T x-T y, x-y] \geq r\|x-y\|^{2}, \forall x, y \in X ;
$$

(v) $r$-strongly monotone with respect to $A$ if there exists a constant $r>0$ such that

$$
[T x-T y, A x-A y] \geq r\|x-y\|^{2}, \forall x, y \in X ;
$$

(vi) $m$-relaxed monotone if there exists a constant $m>0$ such that

$$
[T x-T y, x-y] \geq(-m)\|x-y\|^{2}, \forall x, y \in X ;
$$

(vii) $m$-relaxed monotone with respect to $A$ if there exists a constant $m>0$ such that

$$
[T x-T y, A x-A y] \geq(-m)\|x-y\|^{2}, \forall x, y \in X ;
$$

(viii) $(c, \mu)$-relaxed cocoercive if there exist constant $c, \mu>0$ such that

$$
[T x-T y, x-y] \geq(-c)\|T x-T y\|^{2}+\mu\|x-y\|^{2}, \forall x, y \in X ;
$$


(ix) $(c, \mu)$-relaxed cocoercive with respect to $A$ if there exist constant $c, \mu>0$ such that

$$
[T x-T y, A x-A y] \geq(-c)\|T x-T y\|^{2}+\mu\|x-y\|^{2}, \forall x, y \in X ;
$$

(x) $s$-Lipschitz continuous if there exists a constant $s>0$ such that

$$
\|T x-T y\| \leq s\|x-y\|, \forall x, y \in X .
$$

In the following example we see that a map is not monotone but it is monotone with respect to another operator.

\section{Example 2.1}

Let $T: \mathbb{R} \rightarrow \mathbb{R}$ be a map defined by $T(x)=-x$ and $A: \mathbb{R} \rightarrow \mathbb{R}$ be defined by $A(x)=-\frac{1}{2} x$. One can easily see that $T$ is not monotone, but it is monotone with respect to $A$.

\section{Definition 2.2}

A single valued map $\eta: X \times X \rightarrow X$ is said to be $\tau$-Lipschitz continuous if there exists a constant $\tau>0$ such that $\|\eta(x, y)\| \leq \tau\|x-y\|$ for all $x, y \in X$.

Let $M: X \rightarrow 2^{X}$ be a set valued map. We denote both the mapping and its graph by $M$, that is $M=\{(x, y)$ : $y \in M(x)\}$. The domain of $M$ is defined by

$$
D(M)=\{x \in X: \exists y \in X:(x, y) \in M\} .
$$

The range of $M$ is defined by

$$
R(M)=\{y \in X: \exists x \in X:(x, y) \in M\} .
$$

The inverse $M^{-1}$ of $M$ is $\{(y, x):(x, y) \in M\}$.

For any two set valued mappings $N, M: X \rightarrow 2^{X}$ and any real number $\rho$, we define

$$
\begin{aligned}
& N+M=\{(x, y+z):(x, y) \in N,(x, z) \in M\}, \\
& \rho M=\{(x, \rho y):(x, y) \in M\} .
\end{aligned}
$$

For a map $A: X \rightarrow X$ and a set valued map $M: X \rightarrow 2^{X}$, we define

$$
A+M=\{(x, y+z): A x=y \text { and }(x, z) \in M\} .
$$

Definition 2.3

Let $\eta: X \times X \rightarrow X$ and $A: X \rightarrow X$ be single valued mappings. Let $M: X \rightarrow 2^{X}$ be a set valued map. Then the map $M$ is said to be:

(i) monotone if $[u-v, x-y] \geq 0$ for all $(x, u) \in M,(y, v) \in M$;

(ii) monotone with respect to $A$ if $[u-v, A x-A y] \geq 0$ for all $(x, u) \in M,(y, v) \in M$;

(iii) $\eta$-monotone if $[u-v, \eta(x, y)] \geq 0$ for all $(x, u) \in M,(y, v) \in M$;

(iv) $\eta$-monotone with respect to $A$ if $[u-v, \eta(A x, A y)] \geq 0$ for all $(x, u) \in M,(y, v) \in M$;

(v) strictly $\eta$-monotone if $M$ is $\eta$-monotone and equality holds if and only if $x=y$;

(vi) $(r, \eta)$-strongly monotone if there exists a constant $r>0$ such that $[u-v, \eta(x, y)] \geq r\|x-y\|^{2}$ for all $(x, u) \in$ $M,(y, v) \in M$;

(vii) $(r, \eta)$-strongly monotone with respect to $A$ if there exists a constant $r>0$ such that $[u-v, \eta(A x, A y)] \geq$ $r\|x-y\|^{2}$ for all $(x, u) \in M,(y, v) \in M$;

(viii) $(m, \eta)$-relaxed monotone if there exists a constant $m>0$ such that $[u-v, \eta(x, y)] \geq(-m)\|x-y\|^{2}$ for all $(x, u) \in M,(y, v) \in M$;

(ix) $(m, \eta)$-relaxed monotone with respect to $A$ if there exists a constant $m>0$ such that $[u-v, \eta(A x, A y)] \geq$ $(-m)\|x-y\|^{2}$ for all $(x, u) \in M,(y, v) \in M$;

(x) maximal monotone if $M$ is monotone and $(I+\rho M)(X)=X$ for all $\rho>0$, where $I$ is the identity mapping on $X$;

(xi) maximal $\eta$-monotone if $M$ is $\eta$-monotone and $(I+\rho M)(X)=X$ for all $\rho>0$. 
Definition 2.4

Let $A: X \rightarrow X, H: X \rightarrow X$ and $\eta: X \times X \rightarrow X$ be single valued mappings. Let $M: X \rightarrow 2^{X}$ be a set valued map. Then $M$ is said to be:

(i) $A$-maximal $m$-relaxed monotone if $M$ is $m$-relaxed monotone and $(A+\rho M)(X)=X$ for all $\rho>0$;

(ii) Relative $A$-maximal $m$-relaxed monotone if $M$ is $m$-relaxed monotone with respect to $A$ and $(A+\rho M)(X)=$ $X$ for all $\rho>0$;

(iii) $(A, \eta)$-maximal $m$-relaxed monotone if $M$ is $(m, \eta)$-relaxed monotone and $(A+\rho M)(X)=X$ for all $\rho>0$;

(iv) Relative $(A, \eta)$-maximal $m$-relaxed monotone if $M$ is $(m, \eta)$-relaxed monotone with respect to $A$ and $(A+\rho M)(X)=X$ for all $\rho>0$;

(v) $H$-maximal monotone if $M$ is monotone and $(H+\rho M)(X)=X$ for all $\rho>0$;

(vi) $(H, \eta)$-maximal monotone if $M$ is $\eta$-monotone and $(H+\rho M)(X)=X$ for all $\rho>0$.

Lemma 2.1

Let $\eta: X \times X \rightarrow X$ be a single valued mapping, $A: X \rightarrow X$ be a strictly $\eta$-monotone mapping, and $M: X \rightarrow 2^{X}$ be an $(A, \eta)$-maximal $m$-relaxed monotone mapping. Then the mapping $(A+\rho M)^{-1}$ is single valued.

Proof

For a given $u \in X$, assume that $x, y \in(A+\rho M)^{-1}(u)$. Then we have

$$
\frac{1}{\rho}(u-A(x)) \in M(x) ; \quad \frac{1}{\rho}(u-A(y)) \in M(y) .
$$

Since $M$ is $(m, \eta)$-relaxed monotone, it follows that

$$
\begin{aligned}
& \frac{1}{\rho}[u-A(x)-(u-A(y)), \eta(x, y)] \geq(-m)\|x-y\|^{2} \\
& \Rightarrow[A x-A y, \eta(x, y)] \leq(\rho m)\|x-y\|^{2} .
\end{aligned}
$$

Again $A$ is strictly $\eta$-monotone, that is $[A x-A y, \eta(x, y)] \geq 0, \forall x, y \in X$ and and equality hold if and only if $x=y$. Hence we have

$$
0 \leq[A x-A y, \eta(x, y)] \leq(\rho m)\|x-y\|^{2}
$$

This is possible only when $x=y$, since $\rho$ and $m$ are positive. Hence one can conclude that the operator $(A+\rho M)^{-1}$ is single valued.

Now we can define the generalized resolvent operator associated with $(A, \eta)$-maximal $m$-relaxed monotone mapping.

\section{Definition 2.5}

Let $\eta: X \times X \rightarrow X$ be a single valued mapping, $A: X \rightarrow X$ be a strictly $\eta$-monotone mapping, and $M: X \rightarrow 2^{X}$ be an $(A, \eta)$-maximal $m$-relaxed monotone mapping. Then the generalized resolvent operator $J_{\rho, M}^{A, \eta}: X \rightarrow X$ is defined by

$$
J_{\rho, M}^{A, \eta}(x)=(A+\rho M)^{-1}(x) \text { for all } x \in X
$$

where $\rho$ is a positive constant.

\section{Lemma 2.2}

Let $\eta: X \times X \rightarrow X$ be a $\tau$-Lipschitz continuous map, $A: X \rightarrow X$ be an $(r, \eta)$-strongly monotone mapping and $M: X \rightarrow 2^{X}$ be an $(A, \eta)$-maximal $m$-relaxed monotone mapping. Then the generalized resolvent operator $J_{\rho, M}^{A, \eta}: X \rightarrow X$ is $\frac{\tau}{r-\rho m}$-Lipschitz continuous for $0<\rho<\frac{r}{m}$. 
Proof

For any $x, y \in X$, we have

$$
\begin{aligned}
& J_{\rho, M}^{A, \eta}(x)=(A+\rho M)^{-1}(x) \\
& J_{\rho, M}^{A, \eta}(y)=(A+\rho M)^{-1}(y) .
\end{aligned}
$$

This implies that

$$
\begin{aligned}
& \frac{1}{\rho}\left\{x-A J_{\rho, M}^{A, \eta}(x)\right\} \in M J_{\rho, M}^{A, \eta}(x) \\
& \frac{1}{\rho}\left\{y-A J_{\rho, M}^{A, \eta}(y)\right\} \in M J_{\rho, M}^{A, \eta}(y) .
\end{aligned}
$$

Since $M$ is $(A, \eta)$-maximal $m$-relaxed monotone, we have

$$
\begin{aligned}
& \frac{1}{\rho}\left[x-A J_{\rho, M}^{A, \eta}(x)-\left\{y-A J_{\rho, M}^{A, \eta}(y)\right\}, \eta\left(J_{\rho, M}^{A, \eta}(x), J_{\rho, M}^{A, \eta}(y)\right)\right] \\
= & \frac{1}{\rho}\left[x-y-\left\{A J_{\rho, M}^{A, \eta}(x)-A J_{\rho, M}^{A, \eta}(y)\right\}, \eta\left(J_{\rho, M}^{A, \eta}(x), J_{\rho, M}^{A, \eta}(y)\right)\right] \\
\geq & (-m)\left\|J_{\rho, M}^{A, \eta}(x)-J_{\rho, M}^{A, \eta}(y)\right\|^{2} .
\end{aligned}
$$

Again using the above inequality and $(r, \eta)$-strong monotonicity of $A$, we get

$$
\begin{aligned}
& \|x-y\|\left\|\eta\left(J_{\rho, M}^{A, \eta}(x), J_{\rho, M}^{A, \eta}(y)\right)\right\| \\
\geq & {\left[x-y, \eta\left(J_{\rho, M}^{A, \eta}(x), J_{\rho, M}^{A, \eta}(y)\right)\right] } \\
= & {\left[x-y-\left\{A J_{\rho, M}^{A, \eta}(x)-A J_{\rho, M}^{A, \eta}(y)\right\}, \eta\left(J_{\rho, M}^{A, \eta}(x), J_{\rho, M}^{A, \eta}(y)\right)\right] } \\
& +\left[A J_{\rho, M}^{A, \eta}(x)-A J_{\rho, M}^{A, \eta}(y), \eta\left(J_{\rho, M}^{A, \eta}(x), J_{\rho, M}^{A, \eta}(y)\right)\right] \\
\geq & -\rho m\left\|J_{\rho, M}^{A, \eta}(x)-J_{\rho, M}^{A, \eta}(y)\right\|^{2}+r\left\|J_{\rho, M}^{A, \eta}(x)-J_{\rho, M}^{A, \eta}(y)\right\|^{2} \\
= & (r-\rho m)\left\|J_{\rho, M}^{A, \eta}(x)-J_{\rho, M}^{A, \eta}(y)\right\|^{2} .
\end{aligned}
$$

Applying the $\tau$-Lipschitz continuity of $\eta$ in the above inequality, we get

$$
\begin{aligned}
\|x-y\| \tau\left\|J_{\rho, M}^{A, \eta}(x)-J_{\rho, M}^{A, \eta}(y)\right\| & \geq\|x-y\|\left\|\eta\left(J_{\rho, M}^{A, \eta}(x), J_{\rho, M}^{A, \eta}(y)\right)\right\| \\
& \geq(r-\rho m)\left\|J_{\rho, M}^{A, \eta}(x)-J_{\rho, M}^{A, \eta}(y)\right\|^{2} .
\end{aligned}
$$

This implies that

$$
\Rightarrow\left\|J_{\rho, M}^{A, \eta}(x)-J_{\rho, M}^{A, \eta}(y)\right\| \leq \frac{\tau}{r-\rho m}\|x-y\|, \text { where } 0<\rho<\frac{r}{m} .
$$

\section{Lemma 2.3}

Let $\eta: X \times X \rightarrow X$ be $t$-strongly monotone and $\tau$-Lipschitz continuous, $A: X \rightarrow X$ be $r$-strongly monotone, and let $M: X \rightarrow 2^{X}$ be a relative $(A, \eta)$-maximal monotone mapping. Then the resolvent operator $J_{\rho, M}^{A, \eta}: X \rightarrow X$ is $\frac{\tau}{r t}$-Lipschitz continuous.

Proof

For any $x, y \in X$, we have

$$
\begin{aligned}
& J_{\rho, M}^{A, \eta}(x)=(A+\rho M)^{-1}(x) \\
& J_{\rho, M}^{A, \eta}(y)=(A+\rho M)^{-1}(y) .
\end{aligned}
$$


This implies that

$$
\begin{aligned}
& \frac{1}{\rho}\left\{x-A J_{\rho, M}^{A, \eta}(x)\right\} \in M J_{\rho, M}^{A, \eta}(x) \\
& \frac{1}{\rho}\left\{y-A J_{\rho, M}^{A, \eta}(y)\right\} \in M J_{\rho, M}^{A, \eta}(y) .
\end{aligned}
$$

Since $M$ is $\eta$-monotone with respect to $A$, we have

$$
\frac{1}{\rho}\left[x-y-\left\{A J_{\rho, M}^{A, \eta}(x)-A J_{\rho, M}^{A, \eta}(y)\right\}, \eta\left(A J_{\rho, M}^{A, \eta}(x), A J_{\rho, M}^{A, \eta}(y)\right)\right] \geq 0 .
$$

Using the $t$-strongly monotonicity of $\eta$ in the above inequality, we get

$$
\begin{aligned}
{\left[x-y, \eta\left(A J_{\rho, M}^{A, \eta}(x), A J_{\rho, M}^{A, \eta}(y)\right)\right] } & \geq\left[A J_{\rho, M}^{A, \eta}(x)-A J_{\rho, M}^{A, \eta}(y), \eta\left(A J_{\rho, M}^{A, \eta}(x), A J_{\rho, M}^{A, \eta}(y)\right)\right] \\
& \geq t\left\|A J_{\rho, M}^{A, \eta}(x)-A J_{\rho, M}^{A, \eta}(y)\right\|^{2} .
\end{aligned}
$$

Again using the $\tau$-Lipschitz continuity of $\eta$ in the above inequality, we get

$$
\begin{aligned}
\|x-y\| \tau\left\|A J_{\rho, M}^{A, \eta}(x)-A J_{\rho, M}^{A, \eta}(y)\right\| & \geq\|x-y\|\left\|\eta\left(A J_{\rho, M}^{A, \eta}(x), A J_{\rho, M}^{A, \eta}(y)\right)\right\| \\
& \geq\left[x-y, \eta\left(A J_{\rho, M}^{A, \eta}(x), A J_{\rho, M}^{A, \eta}(y)\right)\right] \\
& \geq t\left\|A J_{\rho, M}^{A, \eta}(x)-A J_{\rho, M}^{A, \eta}(y)\right\|^{2} . \\
\Rightarrow\left\|A J_{\rho, M}^{A, \eta}(x)-A J_{\rho, M}^{A, \eta}(y)\right\| \leq \frac{\tau}{t}\|x-y\| . &
\end{aligned}
$$

Since $A$ is $r$-strongly monotone, we have

$$
\begin{aligned}
r\left\|J_{\rho, M}^{A, \eta}(x)-J_{\rho, M}^{A, \eta}(y)\right\|^{2} & \leq\left[A J_{\rho, M}^{A, \eta}(x)-A J_{\rho, M}^{A, \eta}(y), J_{\rho, M}^{A, \eta}(x)-J_{\rho, M}^{A, \eta}(y)\right] \\
& \leq\left\|A J_{\rho, M}^{A, \eta}(x)-A J_{\rho, M}^{A, \eta}(y)\right\|\left\|J_{\rho, M}^{A, \eta}(x)-J_{\rho, M}^{A, \eta}(y)\right\| \\
& \leq \frac{\tau}{t}\|x-y\|\left\|J_{\rho, M}^{A, \eta}(x)-J_{\rho, M}^{A, \eta}(y)\right\| . \\
\Rightarrow\left\|J_{\rho, M}^{A, \eta}(x)-J_{\rho, M}^{A, \eta}(y)\right\| \leq \frac{\tau}{r t}\|x-y\| . &
\end{aligned}
$$

As the Lemma 2.1 and Lemma 2.2, we can have the following result for $(H, \eta)$-maximal monotone mapping.

\section{Lemma 2.4}

Let $\eta: X \times X \rightarrow X$ be a single valued mapping, $H: X \rightarrow X$ be a strictly $\eta$-monotone mapping, and $M: X \rightarrow 2^{X}$ be an $(H, \eta)$-maximal monotone mapping. Then the mapping $(H+\rho M)^{-1}$ is single valued.

\section{Definition 2.6}

Let $\eta: X \times X \rightarrow X$ be a single valued mapping, $H: X \rightarrow X$ be a strictly $\eta$-monotone mapping, and $M: X \rightarrow 2^{X}$ be an $(H, \eta)$-maximal monotone mapping. Then the generalized resolvent operator $J_{\rho, M}^{H, \eta}: X \rightarrow X$ is defined by

$$
J_{\rho, M}^{H, \eta}(x)=(H+\rho M)^{-1}(x) \text { for all } x \in X,
$$

where $\rho$ is a positive constant.

\section{Lemma 2.5}

Let $\eta: X \times X \rightarrow X$ be a $\tau$-Lipschitz continuous map, $H: X \rightarrow X$ be an $(r, \eta)$-strongly monotone mapping and $M: X \rightarrow 2^{X}$ be an $(H, \eta)$-maximal monotone mapping. Then the generalized resolvent operator $J_{\rho, M}^{H, \eta}: X \rightarrow X$ is $\frac{\tau}{r}$-Lipschitz continuous. 
Housdorff pseudo-metric $\mathcal{D}: 2^{X} \times 2^{X} \rightarrow \mathbb{R} \cup\{+\infty\}$ is defined as

$$
\mathcal{D}(A, B)=\max \left\{\sup _{x \in A} \inf _{y \in B}\|x-y\|, \sup _{x \in B} \inf _{y \in A}\|x-y\|\right\}
$$

for that any two subsets $A$ and $B$ of $X$. When the domain $2^{X}$ is restricted to the closed bounded subsets of $X$, then $\mathcal{D}$ is the Housdorff metric.

Definition 2.7

A set valued map $M: X \rightarrow 2^{X}$ is said to be $\mathcal{D}$-Lipschitz continuous if there exists a constant $\nu>0$ such that

$$
\mathcal{D}(M(x), M(y)) \leq \nu\|x-y\| \text { for all } x, y \in X .
$$

\section{System of nonlinear variational inclusion problems}

In this section we state the problem that we are intended to solve and discuss its various forms. We consider the following general system of nonlinear variational inclusion problems involving $(A, \eta)$-maximal $m$-relaxed monotone mappings.

Let $X_{1}$ and $X_{2}$ be 2-uniformly smooth real Banach spaces, and $K_{1}$ and $K_{2}$ be two nonempty, closed and convex subsets $X_{1}$ and $X_{2}$, respectively. Let $F: X_{1} \times X_{2} \rightarrow X_{1}, G: X_{1} \times X_{2} \rightarrow X_{2}, A_{i}: X_{i} \rightarrow X_{i}$ and $\eta_{i}: X_{i} \times X_{i} \rightarrow$ $X_{i}(i=1,2)$ be nonlinear single valued mappings. Let $U: X_{1} \rightarrow 2^{X_{1}}, V: X_{2} \rightarrow 2^{X_{2}}$ be set valued mappings, and let $M_{i}: X_{i} \rightarrow 2^{X_{i}}$ be $\left(A_{i}, \eta_{i}\right)$-maximal $m_{i}$-relaxed monotone mappings $(i=1,2)$. We consider the problem of finding an element $(a, b) \in X_{1} \times X_{2}, u \in U(a), v \in V(b)$ such that

$$
\left\{\begin{array}{l}
0 \in F(a, v)+M_{1}(a) \\
0 \in G(u, b)+M_{2}(b) .
\end{array}\right.
$$

\section{Special Cases:}

Case (I): When $M_{1}(x)=\partial \varphi(x)$ and $M_{2}(y)=\partial \phi(y)$ for all $x \in X_{1}$ and $y \in X_{2}$, where $\varphi: X_{1} \rightarrow \mathbb{R} \cup\{+\infty\}$ and $\phi: X_{2} \rightarrow \mathbb{R} \cup\{+\infty\}$ are two proper, convex and lower semi-continuous functionals, $\partial \varphi$ and $\partial \phi$ are subdifferentials of $\varphi$ and $\phi$, respectively. Then the problem (3.3) reduces to the following problem:

Find $(a, b) \in X_{1} \times X_{2}, u \in U(a), v \in V(b)$ such that

$$
\left\{\begin{array}{l}
{[F(a, v), x-a]+\varphi(x)-\varphi(a) \geq 0, \forall x \in X_{1}} \\
{[G(u, b), y-b]+\phi(y)-\phi(b) \geq 0, \forall y \in X_{2} .}
\end{array}\right.
$$

The above system is called a system of set valued mixed variational inequality problems.

Case (II): If $U$ and $V$ are both identity mappings, then problem (3.4) reduces to the problem:

Find $(a, b) \in X_{1} \times X_{2}$ such that

$$
\left\{\begin{array}{l}
{[F(a, b), x-a]+\varphi(x)-\varphi(a) \geq 0, \forall x \in X_{1}} \\
{[G(a, b), y-b]+\phi(y)-\phi(b) \geq 0, \forall y \in X_{2} .}
\end{array}\right.
$$

The above nonlinear variational inequality problem was studied by Cho et al. [4].

Case (III): If $M_{1}(x)=\partial \delta_{K_{1}}(x)$ and $M_{2}(y)=\partial \delta_{K_{2}}(y)$ for all $x \in K_{1}$ and $y \in K_{2}$, where $K_{1}$ and $K_{2}$ are nonempty closed convex subsets of $X_{1}$ and $X_{2}$, respectively, and $\delta_{K_{1}}$ and $\delta_{K_{2}}$ are indicator functions of $K_{1}$ and $K_{2}$, respectively. Then the problem (3.4) reduces to the following problem:

Find $(a, b) \in \in K_{1} \times K_{2}$ such that

$$
\left\{\begin{array}{l}
{[F(a, b), x-a] \geq 0, \forall x \in K_{1}} \\
{[G(a, b), y-b] \geq 0, \forall y \in K_{2}}
\end{array}\right.
$$




\section{Existence of solution and approximation solvability}

This section delineates about the existence of solution of the proposed variational inclusion problem (3.3). We start with converting the system of variational inclusion problems into fixed point type of problems by taking the help of generalized resolvent operator. Then we prove the existence of solutions for the system. We construct iterative algorithm to approximate the solution of the proposed problem and discuss its convergency.

In the following theorem, an equivalent formulation of the problem (3.3) is given. Through out this section we assume that $X_{1}$ and $X_{2}$ are 2- uniformly smooth real Banach spaces, and $K_{1}$ and $K_{2}$ be two nonempty, closed and convex subsets $X_{1}$ and $X_{2}$, respectively. Let $F: X_{1} \times X_{2} \rightarrow X_{1}, G: X_{1} \times X_{2} \rightarrow X_{2}, A_{i}: X_{i} \rightarrow X_{i}$ and $\eta_{i}: X_{i} \times X_{i} \rightarrow X_{i}(i=1,2)$ be nonlinear single valued mappings. Let $U: X_{1} \rightarrow 2^{X_{1}}, V: X_{2} \rightarrow 2^{X_{2}}$ be set valued mappings, and let $M_{i}: X_{i} \rightarrow 2^{X_{i}}$ be $\left(A_{i}, \eta_{i}\right)$-maximal $m_{i}$-relaxed monotone mappings $(i=1,2)$.

\section{Theorem 4.1}

Let $(a, b) \in X_{1} \times X_{2}, u \in U(a), v \in V(b)$. Then $(a, b, u, v)$ is a solution of the problem (3.3) if and only if $(a, b, u, v)$ satisfies

$$
\left\{\begin{array}{l}
a=J_{\rho_{1}, M_{1}}^{A_{1}, \eta_{1}}\left(A_{1}(a)-\rho_{1} F(a, v)\right), \\
b=J_{\rho_{2}, M_{2}}^{A_{2}, \eta_{2}}\left(A_{2}(a)-\rho_{2} G(u, b)\right),
\end{array}\right.
$$

where $\rho_{1}$ and $\rho_{2}$ are two positive constants.

Proof

Let $(a, b, u, v)$ be a solution of (3.3). That is

$$
\begin{aligned}
& 0 \in F(a, v)+M_{1}(a) \\
& 0 \in G(u, b)+M_{2}(b) .
\end{aligned}
$$

This implies that

$$
\begin{array}{ll} 
& -\rho_{1} F(a, v) \in \rho_{1} M_{1}(a) . \\
\Rightarrow \quad & A_{1}(a)-\rho_{1} F(a, v) \in A_{1}(a)+\rho_{1} M_{1}(a)=\left(A_{1}+\rho_{1} M_{1}\right)(a) . \\
\Rightarrow \quad & J_{\rho_{1}, M_{1}}^{A_{1}, \eta_{1}}\left(A_{1}(a)-\rho_{1} F(a, v)\right)=a .
\end{array}
$$

Similarly, we can show that $J_{\rho_{2}, M_{2}}^{A_{2}, \eta_{2}}\left(A_{2}(a)-\rho_{2} G(u, b)\right)=b$.

Conversely, assume that

$$
\begin{array}{r}
a=J_{\rho_{1}, M_{1}}^{A_{1}, \eta_{1}}\left(A_{1}(a)-\rho_{1} F(a, v)\right), \\
b=J_{\rho_{2}, M_{2}}^{A_{2}, \eta_{2}}\left(A_{2}(a)-\rho_{2} G(u, b)\right) .
\end{array}
$$

This implies that

$$
\begin{aligned}
& \left(A_{1}+\rho_{1} M_{1}\right)(a)=A_{1}(a)-\rho_{1} F(a, v) . \\
\Rightarrow \quad & 0 \in F(a, v)+M_{1}(a) .
\end{aligned}
$$

Similarly, one can show that $0 \in G(u, b)+M_{2}(b)$.

Using the above theorem, we can able to construct the following algorithm:

\section{Algorithm 4.1}

Step 1: Choose initial approximation $\left(a_{0}, b_{0}\right) \in X_{1} \times X_{2}$ and $u_{0} \in U\left(a_{0}\right), v_{0} \in V\left(b_{0}\right)$. 
Step 2: Construct the iteration

$$
\left\{\begin{array}{l}
a_{k+1}=\left(1-\lambda_{k}-\delta_{k}\right) a_{k}+\lambda_{k} J_{\rho_{1}, M_{1}}^{A_{1}, \eta_{1}}\left(A_{1}\left(a_{k}\right)-\rho_{1} F\left(a_{k}, v_{k}\right)\right), \\
b_{k+1}=\left(1-\lambda_{k}-\delta_{k}\right) b_{k}+\lambda_{k} J_{\rho_{2}, M_{2}}^{A_{2}, \eta_{2}}\left(A_{2}\left(b_{k}\right)-\rho_{2} G\left(u_{k}, b_{k}\right)\right)
\end{array}\right.
$$

where $\lambda_{k}$ and $\delta_{k}$ are positive real constant such that $0<\lambda_{k}+\delta_{k} \leq 1$, and $\lim \sup _{k>0} \lambda_{k}<1$.

Step 3: Choose $u_{k+1} \in U\left(a_{k+1}\right)$ and $v_{k+1} \in V\left(b_{k+1}\right)$ such that

$$
\left\{\begin{array}{l}
\left\|u_{k+1}-u_{k}\right\| \leq\left(1+(1+k)^{-1}\right) \mathcal{D}_{1}\left(U\left(a_{k+1}\right), U\left(a_{k}\right)\right) \\
\left\|v_{k+1}-v_{k}\right\| \leq\left(1+(1+k)^{-1}\right) \mathcal{D}_{2}\left(V\left(b_{k+1}\right), V\left(b_{k}\right)\right)
\end{array}\right.
$$

where $\mathcal{D}_{i}(.,$.$) is the Hausdorff pseudo-metric on 2^{X_{i}}$ for $i=1,2$.

Step 4: If $a_{k+1}, b_{k+1}, u_{k+1}, v_{k+1}$ satisfy (4.8) to a sufficient degree of accuracy, then stop; else set $k=k+1$ and return to Step 2.

\section{Theorem 4.2}

Let $\eta_{i}: X_{i} \times X_{i} \rightarrow X_{i}$ be $\tau_{i}$-Lipschitz continuous, $A_{i}: X_{i} \rightarrow X_{i}$ be $\left(r_{i}, \eta_{i}\right)$-strongly monotone and $\beta_{i}$-Lipschitz continuous, and let $M_{i}: X_{i} \rightarrow 2^{X_{i}}$ be $\left(A_{i}, \eta_{i}\right)$-maximal $m_{i}$-relaxed monotone mappings for $i=1,2$. Let $U: X_{1} \rightarrow$ $C\left(X_{1}\right)$ be $\mathcal{D}_{1}-\gamma_{1}$-Lipschitz continuous and $V: X_{2} \rightarrow C\left(X_{2}\right)$ be $\mathcal{D}_{2}-\gamma_{2}$-Lipschitz continuous. Let $F: X_{1} \times$ $X_{2} \rightarrow X_{1}$ be a nonlinear mapping such that for any given $(a, b) \in X_{1} \times X_{2}, F(., b)$ is $\left(c_{1}, \mu_{1}\right)$-relaxed cocoercive with respect to $A_{1}$ and $\alpha_{1}$-Lipschitz continuous, and $F(a,$.$) is \xi_{1}$-Lipschitz continuous. Let $G: X_{1} \times X_{2} \rightarrow X_{2}$ be another nonlinear mapping such that for any given $(x, y) \in X_{1} \times X_{2}, G(x,$.$) is \left(c_{2}, \mu_{2}\right)$-relaxed cocoercive with respect to $A_{2}$ and $\alpha_{2}$-Lipschitz continuous, and $G(., y)$ is $\xi_{2}$-Lipschitz continuous. If there exist positive real constants $\rho_{1}, \rho_{2}$ such that

$$
\left\{\begin{array}{l}
\tau_{1}\left(r_{2}-\rho_{2} m_{2}\right) \sqrt{\beta_{1}^{2}+2 c_{1} \rho_{1} \alpha_{1}^{2}-2 \rho_{1} \mu_{1}+C \rho_{1}^{2} \alpha_{1}^{2}}+\tau_{2}\left(r_{1}-\rho_{1} m_{1}\right) \rho_{2} \xi_{2} \gamma_{1}<e, \\
\tau_{2}\left(r_{1}-\rho_{1} m_{1}\right) \sqrt{\beta_{2}^{2}+2 c_{2} \rho_{2} \alpha_{2}^{2}-2 \rho_{2} \mu_{2}+C \rho_{2}^{2} \alpha_{2}^{2}}+\tau_{1}\left(r_{2}-\rho_{2} m_{2}\right) \rho_{1} \xi_{1} \gamma_{2}<e,
\end{array}\right.
$$

where $e=\left(r_{1}-\rho_{1} m_{1}\right)\left(r_{2}-\rho_{2} m_{2}\right)$. Then the variational inclusion problem (3.3) has a solution $(a, b, u, v)$. The iterative sequences $\left\{a_{k}\right\},\left\{b_{k}\right\},\left\{u_{k}\right\},\left\{v_{k}\right\}$ generated by Algorithm 4.1 converge strongly to the solution $(a, b, u, v)$.

\section{Proof}

Using the Algorithm 4.1 and Lemma 2.2, we have

$$
\begin{aligned}
& \left\|a_{k+1}-a_{k}\right\| \\
= & \|\left(1-\lambda_{k}-\delta_{k}\right) a_{k}+\lambda_{k} J_{\rho_{1}, M_{1}}^{A_{1}, \eta_{1}}\left(A_{1}\left(a_{k}\right)-\rho_{1} F\left(a_{k}, v_{k}\right)\right) \\
& -\left(1-\lambda_{k}-\delta_{k}\right) a_{k-1}+\lambda_{k} J_{\rho_{1}, M_{1}}^{A_{1}, \eta_{1}}\left(A_{1}\left(a_{k-1}\right)-\rho_{1} F\left(a_{k-1}, v_{k-1}\right)\right) \| \\
\leq \quad & \left(1-\lambda_{k}-\delta_{k}\right)\left\|a_{k}-a_{k-1}\right\| \\
& +\lambda_{k}\left\|J_{\rho_{1}, M_{1}}^{A_{1}, \eta_{1}}\left(A_{1}\left(a_{k}\right)-\rho_{1} F\left(a_{k}, v_{k}\right)\right)-J_{\rho_{1}, M_{1}}^{A_{1}, \eta_{1}}\left(A_{1}\left(a_{k-1}\right)-\rho_{1} F\left(a_{k-1}, v_{k-1}\right)\right)\right\| \\
\leq \quad & \left(1-\lambda_{k}-\delta_{k}\right)\left\|a_{k}-a_{k-1}\right\| \\
& +\lambda_{k} \frac{\tau_{1}}{r_{1}-\rho_{1} m_{1}}\left\|A_{1}\left(a_{k}\right)-A_{1}\left(a_{k-1}\right)-\rho_{1}\left(F\left(a_{k}, v_{k}\right)-F\left(a_{k-1}, v_{k-1}\right)\right)\right\| \\
\leq & \left(1-\lambda_{k}\right)\left\|a_{k}-a_{k-1}\right\| \\
& +\lambda_{k} \frac{\tau_{1}}{r_{1}-\rho_{1} m_{1}}\left\|A_{1}\left(a_{k}\right)-A_{1}\left(a_{k-1}\right)-\rho_{1}\left(F\left(a_{k}, v_{k}\right)-F\left(a_{k-1}, v_{k}\right)\right)\right\| \\
& +\lambda_{k} \frac{\tau_{1}}{r_{1}-\rho_{1} m_{1}} \rho_{1}\left\|F\left(a_{k-1}, v_{k}\right)-F\left(a_{k-1}, v_{k-1}\right)\right\| .
\end{aligned}
$$


Similarly, we can estimate

$$
\begin{aligned}
& \left\|b_{k+1}-b_{k}\right\| \\
\leq & \left(1-\lambda_{k}\right)\left\|b_{k}-b_{k-1}\right\| \\
& +\lambda_{k} \frac{\tau_{2}}{r_{2}-\rho_{2} m_{2}}\left\|A_{2}\left(b_{k}\right)-A_{2}\left(b_{k-1}\right)-\rho_{2}\left(G\left(u_{k}, b_{k}\right)-G\left(u_{k}, b_{k-1}\right)\right)\right\| \\
& +\lambda_{k} \frac{\tau_{2}}{r_{2}-\rho_{2} m_{2}} \rho_{2}\left\|G\left(u_{k}, b_{k-1}\right)-G\left(u_{k-1}, b_{k-1}\right)\right\| .
\end{aligned}
$$

It is given that $A_{i}$ are $\beta_{i}$-Lipschitz continuous for $i=1,2, F(., b)$ is $\left(c_{1}, \mu_{1}\right)$-relaxed cocoercive with respect to $A_{1}$ and $\alpha_{1}$-Lipschitz continuous, $G(x,$.$) is \left(c_{2}, \mu_{2}\right)$-relaxed cocoercive with respect to $A_{2}$ and $\alpha_{2}$-Lipschitz continuous. Hence we estimate

$$
\begin{aligned}
& \left\|A_{1}\left(a_{k}\right)-A_{1}\left(a_{k-1}\right)-\rho_{1}\left(F\left(a_{k}, v_{k}\right)-F\left(a_{k-1}, v_{k}\right)\right)\right\|^{2} \\
\leq & \left\|A_{1}\left(a_{k}\right)-A_{1}\left(a_{k-1}\right)\right\|^{2}-2 \rho_{1}\left[F\left(a_{k}, v_{k}\right)-F\left(a_{k-1}, v_{k}\right), A_{1}\left(a_{k}\right)-A_{1}\left(a_{k-1}\right)\right] \\
& +C \rho_{1}^{2}\left\|F\left(a_{k}, v_{k}\right)-F\left(a_{k-1}, v_{k}\right)\right\|^{2} \\
\leq & \beta_{1}^{2}\left\|a_{k}-a_{k-1}\right\|^{2}-2 \rho_{1}\left\{-c_{1}\left\|F\left(a_{k}, v_{k}\right)-F\left(a_{k-1}, v_{k}\right)\right\|^{2}+\mu_{1}\left\|a_{k}-a_{k-1}\right\|^{2}\right\} \\
& +C \rho_{1}^{2} \alpha_{1}^{2}\left\|a_{k}-a_{k-1}\right\|^{2} \\
\leq & \beta_{1}^{2}\left\|a_{k}-a_{k-1}\right\|^{2}+2 c_{1} \rho_{1} \alpha_{1}^{2}\left\|a_{k}-a_{k-1}\right\|^{2}-2 \rho_{1} \mu_{1}\left\|a_{k}-a_{k-1}\right\|^{2}+C \rho_{1}^{2} \alpha_{1}^{2}\left\|a_{k}-a_{k-1}\right\|^{2} \\
= & \left(\beta_{1}^{2}+2 c_{1} \rho_{1} \alpha_{1}^{2}-2 \rho_{1} \mu_{1}+C \rho_{1}^{2} \alpha_{1}^{2}\right)\left\|a_{k}-a_{k-1}\right\|^{2}
\end{aligned}
$$

This implies that

$$
\begin{aligned}
& \left\|A_{1}\left(a_{k}\right)-A_{1}\left(a_{k-1}\right)-\rho_{1}\left(F\left(a_{k}, v_{k}\right)-F\left(a_{k-1}, v_{k}\right)\right)\right\| \\
\leq & \sqrt{\beta_{1}^{2}+2 c_{1} \rho_{1} \alpha_{1}^{2}-2 \rho_{1} \mu_{1}+C \rho_{1}^{2} \alpha_{1}^{2}}\left\|a_{k}-a_{k-1}\right\| .
\end{aligned}
$$

Similarly, we have

$$
\begin{aligned}
& \left\|A_{2}\left(b_{k}\right)-A_{2}\left(b_{k-1}\right)-\rho_{2}\left(G\left(u_{k}, b_{k}\right)-G\left(u_{k}, b_{k-1}\right)\right)\right\| \\
\leq & \sqrt{\beta_{2}^{2}+2 c_{2} \rho_{2} \alpha_{2}^{2}-2 \rho_{2} \mu_{2}+C \rho_{2}^{2} \alpha_{2}^{2}}\left\|b_{k}-b_{k-1}\right\| .
\end{aligned}
$$

Again, since $F(a,$.$) is \xi_{1}$-Lipschitz continuous and $V$ is $\mathcal{D}_{2}-\gamma_{2}$-Lipschitz continuous, we have

$$
\begin{aligned}
\left\|F\left(a_{k-1}, v_{k}\right)-F\left(a_{k-1}, v_{k-1}\right)\right\| & \leq \xi_{1}\left\|v_{k}-v_{k-1}\right\| \\
& \leq \xi_{1}\left(1+n^{-1}\right) \mathcal{D}_{2}\left(V\left(b_{k}, V\left(b_{k-1}\right)\right)\right. \\
& \leq \xi_{1}\left(1+n^{-1}\right) \gamma_{2}\left\|b_{k}-b_{k-1}\right\| .
\end{aligned}
$$

Similarly, since $G(., y)$ is $\xi_{2}$-Lipschitz continuous and $U$ is $\mathcal{D}_{1}-\gamma_{1}$-Lipschitz continuous, we have

$$
\left\|G\left(u_{k}, b_{k-1}\right)-G\left(u_{k-1}, b_{k-1}\right)\right\| \leq \xi_{2}\left(1+n^{-1}\right) \gamma_{1}\left\|a_{k}-a_{k-1}\right\| .
$$

Putting (4.13) and (4.15) in (4.11), we get

$$
\begin{aligned}
& \left\|a_{k+1}-a_{k}\right\| \\
\leq & \left(1-\lambda_{k}\right)\left\|a_{k}-a_{k-1}\right\|+\lambda_{k} \frac{\tau_{1}}{r_{1}-\rho_{1} m_{1}} \sqrt{\beta_{1}^{2}+2 c_{1} \rho_{1} \alpha_{1}^{2}-2 \rho_{1} \mu_{1}+C \rho_{1}^{2} \alpha_{1}^{2}}\left\|a_{k}-a_{k-1}\right\| \\
& +\lambda_{k} \frac{\tau_{1}}{r_{1}-\rho_{1} m_{1}} \rho_{1} \xi_{1}\left(1+n^{-1}\right) \gamma_{2}\left\|b_{k}-b_{k-1}\right\| .
\end{aligned}
$$


Similarly, using (4.14) and (4.16) in (4.12), we get

$$
\begin{aligned}
& \left\|b_{k+1}-b_{k}\right\| \\
\leq & \left(1-\lambda_{k}\right)\left\|b_{k}-b_{k-1}\right\|+\lambda_{k} \frac{\tau_{2}}{r_{2}-\rho_{2} m_{2}} \sqrt{\beta_{2}^{2}+2 c_{2} \rho_{2} \alpha_{2}^{2}-2 \rho_{2} \mu_{2}+C \rho_{2}^{2} \alpha_{2}^{2}}\left\|b_{k}-b_{k-1}\right\| \\
& +\lambda_{k} \frac{\tau_{2}}{r_{2}-\rho_{2} m_{2}} \rho_{2} \xi_{2}\left(1+n^{-1}\right) \gamma_{1}\left\|a_{k}-a_{k-1}\right\| .
\end{aligned}
$$

Hence

$$
\begin{aligned}
& \left\|a_{k+1}-a_{k}\right\|+\left\|b_{k+1}-b_{k}\right\| \\
\leq & \left(1-\lambda_{k}+\lambda_{k} \frac{\tau_{1}}{r_{1}-\rho_{1} m_{1}} \sqrt{\beta_{1}^{2}+2 c_{1} \rho_{1} \alpha_{1}^{2}-2 \rho_{1} \mu_{1}+C \rho_{1}^{2} \alpha_{1}^{2}}\right. \\
& \left.+\lambda_{k} \frac{\tau_{2}}{r_{2}-\rho_{2} m_{2}} \rho_{2} \xi_{2}\left(1+n^{-1}\right) \gamma_{1}\right)\left\|a_{k}-a_{k-1}\right\| \\
& +\left(1-\lambda_{k}+\lambda_{k} \frac{\tau_{2}}{r_{2}-\rho_{2} m_{2}} \sqrt{\beta_{2}^{2}+2 c_{2} \rho_{2} \alpha_{2}^{2}-2 \rho_{2} \mu_{2}+C \rho_{2}^{2} \alpha_{2}^{2}}\right. \\
& \left.+\lambda_{k} \frac{\tau_{1}}{r_{1}-\rho_{1} m_{1}} \rho_{1} \xi_{1}\left(1+n^{-1}\right) \gamma_{2}\right)\left\|b_{k}-b_{k-1}\right\| \\
\leq & \left(1-\lambda_{k}\left(1-\theta_{k}\right)\right)\left(\left\|a_{k}-a_{k-1}\right\|+\left\|b_{k}-b_{k-1}\right\|\right) \\
\leq & \left(1-\triangle\left(1-\theta_{k}\right)\right)\left(\left\|a_{k}-a_{k-1}\right\|+\left\|b_{k}-b_{k-1}\right\|\right),
\end{aligned}
$$

where

$$
\begin{aligned}
& \theta_{k}=\max \left\{\frac{\tau_{1}}{r_{1}-\rho_{1} m_{1}} \sqrt{\beta_{1}^{2}+2 c_{1} \rho_{1} \alpha_{1}^{2}-2 \rho_{1} \mu_{1}+C \rho_{1}^{2} \alpha_{1}^{2}}+\frac{\tau_{2}}{r_{2}-\rho_{2} m_{2}} \rho_{2} \xi_{2}\left(1+n^{-1}\right) \gamma_{1},\right. \\
& \left.\frac{\tau_{2}}{r_{2}-\rho_{2} m_{2}} \sqrt{\beta_{2}^{2}+2 c_{2} \rho_{2} \alpha_{2}^{2}-2 \rho_{2} \mu_{2}+C \rho_{2}^{2} \alpha_{2}^{2}}+\frac{\tau_{1}}{r_{1}-\rho_{1} m_{1}} \rho_{1} \xi_{1}\left(1+n^{-1}\right) \gamma_{2}\right\},
\end{aligned}
$$

and $\triangle=\lim \sup _{k \geq 0} \lambda_{k}<1$.

We see that $\theta_{k} \rightarrow \bar{\theta}$ as $k \rightarrow \infty$, where

$$
\begin{aligned}
& \theta=\max \left\{\frac{\tau_{1}}{r_{1}-\rho_{1} m_{1}} \sqrt{\beta_{1}^{2}+2 c_{1} \rho_{1} \alpha_{1}^{2}-2 \rho_{1} \mu_{1}+C \rho_{1}^{2} \alpha_{1}^{2}}+\frac{\tau_{2}}{r_{2}-\rho_{2} m_{2}} \rho_{2} \xi_{2} \gamma_{1},\right. \\
& \left.\frac{\tau_{2}}{r_{2}-\rho_{2} m_{2}} \sqrt{\beta_{2}^{2}+2 c_{2} \rho_{2} \alpha_{2}^{2}-2 \rho_{2} \mu_{2}+C \rho_{2}^{2} \alpha_{2}^{2}}+\frac{\tau_{1}}{r_{1}-\rho_{1} m_{1}} \rho_{1} \xi_{1} \gamma_{2}\right\} .
\end{aligned}
$$

The assumption (4.10) assures that $0<\theta<1$. Therefore, by (4.19) and $0<\lambda_{k}+\delta_{k} \leq 1$ implies that $\left\{a_{k}\right\}$ and $\left\{b_{k}\right\}$ are both Cauchy sequences. Since the spaces $X_{1}$ and $X_{2}$ are complete, there exist $a \in X_{1}$ and $b \in X_{2}$ such that $a_{k} \rightarrow a$ and $b_{k} \rightarrow b$ as $k \rightarrow \infty$.

Next we prove that $u_{k} \rightarrow u \in U(a)$ and $v_{k} \rightarrow v \in V(b)$ as $k \rightarrow \infty$. The inequalities (4.15) and (4.16) guarantee that $\left\{u_{k}\right\}$ and $\left\{v_{k}\right\}$ are also Cauchy sequences. Therefore there exist $u \in X_{1}$ and $v \in X_{2}$ such that $u_{k} \rightarrow u$ and $v_{k} \rightarrow v$ as $k \rightarrow \infty$. Moreover,

$$
\begin{aligned}
d(u, U(a))=\inf \{\|u-t\|: t \in U(a)\} & \leq\left\|u-u_{k}\right\|+d\left(u_{k}, U(a)\right) \\
& \leq\left\|u-u_{k}\right\|+\mathcal{D}_{1}\left(U\left(a_{k}\right), U(a)\right) \\
& \leq\left\|u-u_{k}\right\|+\xi_{1}\left\|a_{k}-a\right\| \rightarrow 0 .
\end{aligned}
$$

Since $U(a)$ is closed, we have $u \in U(a)$. Similarly, we can show that $v \in V(b)$.

Finally, applying the continuity property of $A_{1}, F, A_{2}, G$ and the continuity of the generalized resolvent operator, we get

$$
\left\{\begin{array}{l}
a=J_{\rho_{1}, M_{1}}^{A_{1}, \eta_{1}}\left(A_{1}(a)-\rho_{1} F(a, v)\right), \\
b=J_{\rho_{2}, M_{2}}^{A_{2}, \eta_{2}}\left(A_{2}(a)-\rho_{2} G(u, b)\right) .
\end{array}\right.
$$

Because of Theorem 4.1, we conclude that $(a, b, u, v)$ is a solution of the variational inclusion problem (3.3). 
If we replace the relaxed cocoercivity condition by strong monotonicity condition on the maps $F(., b)$ and $G(x,$.$) , then we get the following result. We write it in the form of a corollary.$

\section{Corollary 4.1}

Let $\eta_{i}: X_{i} \times X_{i} \rightarrow X_{i}$ be $\tau_{i}$-Lipschitz continuous, $A_{i}: X_{i} \rightarrow X_{i}$ be $\left(r_{i}, \eta_{i}\right)$-strongly monotone and $\beta_{i}$-Lipschitz continuous, and let $M_{i}: X_{i} \rightarrow 2^{X_{i}}$ be $\left(A_{i}, \eta_{i}\right)$-maximal $m_{i}$-relaxed monotone mappings for $i=1,2$. Let $U$ : $X_{1} \rightarrow C\left(X_{1}\right)$ be $\mathcal{D}_{1}-\gamma_{1}$-Lipschitz continuous and $V: X_{2} \rightarrow C\left(X_{2}\right)$ be $\mathcal{D}_{2}-\gamma_{2}$-Lipschitz continuous. Let $F$ : $X_{1} \times X_{2} \rightarrow X_{1}$ be a nonlinear mapping such that for any given $(a, b) \in X_{1} \times X_{2}, F(., b)$ is $\mu_{1}$-strongly monotone with respect to $A_{1}$ and $\alpha_{1}$-Lipschitz continuous, and $F(a,$.$) is \xi_{1}$-Lipschitz continuous. Let $G: X_{1} \times X_{2} \rightarrow X_{2}$ be another nonlinear mapping such that for any given $(x, y) \in X_{1} \times X_{2}, G(x,$.$) is \mu_{2}$-strongly monotone with respect to $A_{2}$ and $\alpha_{2}$-Lipschitz continuous, and $G(., y)$ is $\xi_{2}$-Lipschitz continuous. If there exist positive real constants $\rho_{1}, \rho_{2}$ such that

$$
\left\{\begin{array}{l}
\tau_{1}\left(r_{2}-\rho_{2} m_{2}\right) \sqrt{\beta_{1}^{2}-2 \rho_{1} \mu_{1}+C \rho_{1}^{2} \alpha_{1}^{2}}+\tau_{2}\left(r_{1}-\rho_{1} m_{1}\right) \rho_{2} \xi_{2} \gamma_{1}<e, \\
\tau_{2}\left(r_{1}-\rho_{1} m_{1}\right) \sqrt{\beta_{2}^{2}-2 \rho_{2} \mu_{2}+C \rho_{2}^{2} \alpha_{2}^{2}}+\tau_{1}\left(r_{2}-\rho_{2} m_{2}\right) \rho_{1} \xi_{1} \gamma_{2}<e,
\end{array}\right.
$$

where $e=\left(r_{1}-\rho_{1} m_{1}\right)\left(r_{2}-\rho_{2} m_{2}\right)$. Then the variational inclusion problem (3.3) has a solution $(a, b, u, v)$. The iterative sequences $\left\{a_{k}\right\},\left\{b_{k}\right\},\left\{u_{k}\right\},\left\{v_{k}\right\}$ generated by Algorithm 4.1 converge strongly to the solution $(a, b, u, v)$.

In the following theorem we impose the relative $\left(A_{i}, \eta_{i}\right)$-maximal monotonicity on the set valued map $M$. The proof is similar as that of Theorem 4.5, but as the relax condition on $M$ is removed and different conditions on $\eta$ and $A$ are imposed, so we include the proof.

\section{Theorem 4.3}

Let $\eta_{i}: X_{i} \times X_{i} \rightarrow X_{i}$ be $t_{i}$-strongly monotone and $\tau_{i}$-Lipschitz continuous, $A_{i}: X_{i} \rightarrow X_{i}$ be $r_{i}$-strongly monotone and $\beta_{i}$-Lipschitz continuous, and let $M_{i}: X_{i} \rightarrow 2^{X_{i}}$ be relative $\left(A_{i}, \eta_{i}\right)$-maximal monotone mappings for $i=1,2$. Let $U: X_{1} \rightarrow C\left(X_{1}\right)$ be $\mathcal{D}_{1}-\gamma_{1}$-Lipschitz continuous and $V: X_{2} \rightarrow C\left(X_{2}\right)$ be $\mathcal{D}_{2}-\gamma_{2}$-Lipschitz continuous. Let $F: X_{1} \times X_{2} \rightarrow X_{1}$ be a nonlinear mapping such that for any given $(a, b) \in X_{1} \times X_{2}, F(., b)$ is $\left(c_{1}, \mu_{1}\right)$-relaxed cocoercive with respect to $A_{1}$ and $\alpha_{1}$-Lipschitz continuous, and $F(a,$.$) is \xi_{1}$-Lipschitz continuous. Let $G: X_{1} \times X_{2} \rightarrow X_{2}$ be another nonlinear mapping such that for any given $(x, y) \in X_{1} \times X_{2}, G(x,$.$) is \left(c_{2}, \mu_{2}\right)$ relaxed cocoercive with respect to $A_{2}$ and $\alpha_{2}$-Lipschitz continuous, and $G(., y)$ is $\xi_{2}$-Lipschitz continuous. If there exist positive real constants $\rho_{1}, \rho_{2}$ such that

$$
\left\{\begin{array}{l}
\tau_{1} r_{2} t_{2} \sqrt{\beta_{1}^{2}+2 c_{1} \rho_{1} \alpha_{1}^{2}-2 \rho_{1} \mu_{1}+C \rho_{1}^{2} \alpha_{1}^{2}}+\tau_{2} r_{1} t_{1} \rho_{2} \xi_{2} \gamma_{1}<r_{1} r_{2} t_{1} t_{2}, \\
\tau_{2} r_{1} t_{1} \sqrt{\beta_{2}^{2}+2 c_{2} \rho_{2} \alpha_{2}^{2}-2 \rho_{2} \mu_{2}+C \rho_{2}^{2} \alpha_{2}^{2}}+\tau_{1} r_{2} t_{2} \rho_{1} \xi_{1} \gamma_{2}<r_{1} r_{2} t_{1} t_{2}
\end{array}\right.
$$

Then the variational inclusion problem (3.3) has a solution $(a, b, u, v)$. The iterative sequences $\left\{a_{k}\right\},\left\{b_{k}\right\},\left\{u_{k}\right\},\left\{v_{k}\right\}$ generated by Algorithm 4.1 converge strongly to the solution $(a, b, u, v)$. 
Proof

Using the Algorithm 4.1 and Lemma 2.3, we have

$$
\begin{aligned}
& \left\|a_{k+1}-a_{k}\right\| \\
= & \|\left(1-\lambda_{k}-\delta_{k}\right) a_{k}+\lambda_{k} J_{\rho_{1}, M_{1}}^{A_{1}, \eta_{1}}\left(A_{1}\left(a_{k}\right)-\rho_{1} F\left(a_{k}, v_{k}\right)\right) \\
& -\left(1-\lambda_{k}-\delta_{k}\right) a_{k-1}+\lambda_{k} J_{\rho_{1}, M_{1}}^{A_{1}, \eta_{1}}\left(A_{1}\left(a_{k-1}\right)-\rho_{1} F\left(a_{k-1}, v_{k-1}\right)\right) \| \\
\leq \quad & \left(1-\lambda_{k}-\delta_{k}\right)\left\|a_{k}-a_{k-1}\right\| \\
& +\lambda_{k}\left\|J_{\rho_{1}, M_{1}}^{A_{1}, \eta_{1}}\left(A_{1}\left(a_{k}\right)-\rho_{1} F\left(a_{k}, v_{k}\right)\right)-J_{\rho_{1}, M_{1}}^{A_{1}, \eta_{1}}\left(A_{1}\left(a_{k-1}\right)-\rho_{1} F\left(a_{k-1}, v_{k-1}\right)\right)\right\| \\
\leq \quad & \left(1-\lambda_{k}-\delta_{k}\right)\left\|a_{k}-a_{k-1}\right\| \\
& +\lambda_{k} \frac{\tau_{1}}{r_{1} t_{1}}\left\|A_{1}\left(a_{k}\right)-A_{1}\left(a_{k-1}\right)-\rho_{1}\left(F\left(a_{k}, v_{k}\right)-F\left(a_{k-1}, v_{k-1}\right)\right)\right\| \\
\leq \quad & \left(1-\lambda_{k}\right)\left\|a_{k}-a_{k-1}\right\| \\
& +\lambda_{k} \frac{\tau_{1}}{r_{1} t_{1}}\left\|A_{1}\left(a_{k}\right)-A_{1}\left(a_{k-1}\right)-\rho_{1}\left(F\left(a_{k}, v_{k}\right)-F\left(a_{k-1}, v_{k}\right)\right)\right\| \\
& +\lambda_{k} \frac{\tau_{1}}{r_{1} t_{1}} \rho_{1}\left\|F\left(a_{k-1}, v_{k}\right)-F\left(a_{k-1}, v_{k-1}\right)\right\| .
\end{aligned}
$$

Similarly, we can estimate

$$
\begin{aligned}
& \left\|b_{k+1}-b_{k}\right\| \\
\leq & \left(1-\lambda_{k}\right)\left\|b_{k}-b_{k-1}\right\| \\
& +\lambda_{k} \frac{\tau_{2}}{r_{2} t_{2}}\left\|A_{2}\left(b_{k}\right)-A_{2}\left(b_{k-1}\right)-\rho_{2}\left(G\left(u_{k}, b_{k}\right)-G\left(u_{k}, b_{k-1}\right)\right)\right\| \\
& +\lambda_{k} \frac{\tau_{2}}{r_{2} t_{2}} \rho_{2}\left\|G\left(u_{k}, b_{k-1}\right)-G\left(u_{k-1}, b_{k-1}\right)\right\| .
\end{aligned}
$$

It is given that $A_{i}$ are $\beta_{i}$-Lipschitz continuous for $i=1,2, F(., b)$ is $\left(c_{1}, \mu_{1}\right)$-relaxed cocoercive with respect to $A_{1}$ and $\alpha_{1}$-Lipschitz continuous, $G(x,$.$) is \left(c_{2}, \mu_{2}\right)$-relaxed cocoercive with respect to $A_{2}$ and $\alpha_{2}$-Lipschitz continuous. Hence we estimate

$$
\begin{aligned}
& \left\|A_{1}\left(a_{k}\right)-A_{1}\left(a_{k-1}\right)-\rho_{1}\left(F\left(a_{k}, v_{k}\right)-F\left(a_{k-1}, v_{k}\right)\right)\right\|^{2} \\
\leq & \left\|A_{1}\left(a_{k}\right)-A_{1}\left(a_{k-1}\right)\right\|^{2}-2 \rho_{1}\left[F\left(a_{k}, v_{k}\right)-F\left(a_{k-1}, v_{k}\right), A_{1}\left(a_{k}\right)-A_{1}\left(a_{k-1}\right)\right] \\
& +C \rho_{1}^{2}\left\|F\left(a_{k}, v_{k}\right)-F\left(a_{k-1}, v_{k}\right)\right\|^{2} \\
\leq & \beta_{1}^{2}\left\|a_{k}-a_{k-1}\right\|^{2}-2 \rho_{1}\left\{-c_{1}\left\|F\left(a_{k}, v_{k}\right)-F\left(a_{k-1}, v_{k}\right)\right\|^{2}+\mu_{1}\left\|a_{k}-a_{k-1}\right\|^{2}\right\} \\
& +C \rho_{1}^{2} \alpha_{1}^{2}\left\|a_{k}-a_{k-1}\right\|^{2} \\
\leq & \beta_{1}^{2}\left\|a_{k}-a_{k-1}\right\|^{2}+2 c_{1} \rho_{1} \alpha_{1}^{2}\left\|a_{k}-a_{k-1}\right\|^{2}-2 \rho_{1} \mu_{1}\left\|a_{k}-a_{k-1}\right\|^{2}+C \rho_{1}^{2} \alpha_{1}^{2}\left\|a_{k}-a_{k-1}\right\|^{2} \\
= & \left(\beta_{1}^{2}+2 c_{1} \rho_{1} \alpha_{1}^{2}-2 \rho_{1} \mu_{1}+C \rho_{1}^{2} \alpha_{1}^{2}\right)\left\|a_{k}-a_{k-1}\right\|^{2}
\end{aligned}
$$

This implies that

$$
\begin{aligned}
& \left\|A_{1}\left(a_{k}\right)-A_{1}\left(a_{k-1}\right)-\rho_{1}\left(F\left(a_{k}, v_{k}\right)-F\left(a_{k-1}, v_{k}\right)\right)\right\| \\
\leq & \sqrt{\beta_{1}^{2}+2 c_{1} \rho_{1} \alpha_{1}^{2}-2 \rho_{1} \mu_{1}+C \rho_{1}^{2} \alpha_{1}^{2}}\left\|a_{k}-a_{k-1}\right\| .
\end{aligned}
$$

Similarly, we have

$$
\begin{aligned}
& \left\|A_{2}\left(b_{k}\right)-A_{2}\left(b_{k-1}\right)-\rho_{2}\left(G\left(u_{k}, b_{k}\right)-G\left(u_{k}, b_{k-1}\right)\right)\right\| \\
\leq & \sqrt{\beta_{2}^{2}+2 c_{2} \rho_{2} \alpha_{2}^{2}-2 \rho_{2} \mu_{2}+C \rho_{2}^{2} \alpha_{2}^{2}}\left\|b_{k}-b_{k-1}\right\| .
\end{aligned}
$$


Again, since $F(a,$.$) is \xi_{1}$-Lipschitz continuous and $V$ is $\mathcal{D}_{2}-\gamma_{2}$-Lipschitz continuous, we have

$$
\begin{aligned}
\left\|F\left(a_{k-1}, v_{k}\right)-F\left(a_{k-1}, v_{k-1}\right)\right\| & \leq \xi_{1}\left\|v_{k}-v_{k-1}\right\| \\
& \leq \xi_{1}\left(1+n^{-1}\right) \mathcal{D}_{2}\left(V\left(b_{k}, V\left(b_{k-1}\right)\right)\right. \\
& \leq \xi_{1}\left(1+n^{-1}\right) \gamma_{2}\left\|b_{k}-b_{k-1}\right\| .
\end{aligned}
$$

Similarly, since $G(., y)$ is $\xi_{2}$-Lipschitz continuous and $U$ is $\mathcal{D}_{1}-\gamma_{1}$-Lipschitz continuous, we have

$$
\left\|G\left(u_{k}, b_{k-1}\right)-G\left(u_{k-1}, b_{k-1}\right)\right\| \leq \xi_{2}\left(1+n^{-1}\right) \gamma_{1}\left\|a_{k}-a_{k-1}\right\| .
$$

Putting (4.24) and (4.26) in (4.22), we get

$$
\begin{aligned}
& \left\|a_{k+1}-a_{k}\right\| \\
\leq \quad & \left(1-\lambda_{k}\right)\left\|a_{k}-a_{k-1}\right\|+\lambda_{k} \frac{\tau_{1}}{r_{1} t_{1}} \sqrt{\beta_{1}^{2}+2 c_{1} \rho_{1} \alpha_{1}^{2}-2 \rho_{1} \mu_{1}+C \rho_{1}^{2} \alpha_{1}^{2}}\left\|a_{k}-a_{k-1}\right\| \\
& +\lambda_{k} \frac{\tau_{1}}{r_{1} t_{1}} \rho_{1} \xi_{1}\left(1+n^{-1}\right) \gamma_{2}\left\|b_{k}-b_{k-1}\right\| .
\end{aligned}
$$

Similarly, using (4.25) and (4.27) in (4.23), we get

$$
\begin{aligned}
& \left\|b_{k+1}-b_{k}\right\| \\
\leq & \left(1-\lambda_{k}\right)\left\|b_{k}-b_{k-1}\right\|+\lambda_{k} \frac{\tau_{2}}{r_{2} t_{2}} \sqrt{\beta_{2}^{2}+2 c_{2} \rho_{2} \alpha_{2}^{2}-2 \rho_{2} \mu_{2}+C \rho_{2}^{2} \alpha_{2}^{2}}\left\|b_{k}-b_{k-1}\right\| \\
& +\lambda_{k} \frac{\tau_{2}}{r_{2} t_{2}} \rho_{2} \xi_{2}\left(1+n^{-1}\right) \gamma_{1}\left\|a_{k}-a_{k-1}\right\| .
\end{aligned}
$$

Hence

$$
\begin{aligned}
& \left\|a_{k+1}-a_{k}\right\|+\left\|b_{k+1}-b_{k}\right\| \\
\leq \quad & \left(1-\lambda_{k}+\lambda_{k} \frac{\tau_{1}}{r_{1} t_{1}} \sqrt{\beta_{1}^{2}+2 c_{1} \rho_{1} \alpha_{1}^{2}-2 \rho_{1} \mu_{1}+C \rho_{1}^{2} \alpha_{1}^{2}}\right. \\
& \left.+\lambda_{k} \frac{\tau_{2}}{r_{2} t_{2}} \rho_{2} \xi_{2}\left(1+n^{-1}\right) \gamma_{1}\right)\left\|a_{k}-a_{k-1}\right\| \\
& +\left(1-\lambda_{k}+\lambda_{k} \frac{\tau_{2}}{r_{2} t_{2}} \sqrt{\beta_{2}^{2}+2 c_{2} \rho_{2} \alpha_{2}^{2}-2 \rho_{2} \mu_{2}+C \rho_{2}^{2} \alpha_{2}^{2}}\right. \\
& \left.+\lambda_{k} \frac{\tau_{1}}{r_{1} t_{1}} \rho_{1} \xi_{1}\left(1+n^{-1}\right) \gamma_{2}\right)\left\|b_{k}-b_{k-1}\right\| \\
\leq & \left(1-\lambda_{k}\left(1-\theta_{k}\right)\right)\left(\left\|a_{k}-a_{k-1}\right\|+\left\|b_{k}-b_{k-1}\right\|\right) \\
\leq & \left(1-\triangle\left(1-\theta_{k}\right)\right)\left(\left\|a_{k}-a_{k-1}\right\|+\left\|b_{k}-b_{k-1}\right\|\right),
\end{aligned}
$$

where

$$
\begin{aligned}
\theta_{k}= & \max \left\{\frac{\tau_{1}}{r_{1} t_{1}} \sqrt{\beta_{1}^{2}+2 c_{1} \rho_{1} \alpha_{1}^{2}-2 \rho_{1} \mu_{1}+C \rho_{1}^{2} \alpha_{1}^{2}}+\frac{\tau_{2}}{r_{2} t_{2}} \rho_{2} \xi_{2}\left(1+n^{-1}\right) \gamma_{1},\right. \\
& \left.\frac{\tau_{2}}{r_{2} t_{2}} \sqrt{\beta_{2}^{2}+2 c_{2} \rho_{2} \alpha_{2}^{2}-2 \rho_{2} \mu_{2}+C \rho_{2}^{2} \alpha_{2}^{2}}+\frac{\tau_{1}}{r_{1} t_{1}} \rho_{1} \xi_{1}\left(1+n^{-1}\right) \gamma_{2}\right\},
\end{aligned}
$$

and $\triangle=\lim \sup _{k>0} \lambda_{k}<1$.

We see that $\theta_{k} \rightarrow \bar{\theta}$ as $k \rightarrow \infty$, where

$$
\begin{aligned}
& \theta=\max \left\{\frac{\tau_{1}}{r_{1} t_{1}} \sqrt{\beta_{1}^{2}+2 c_{1} \rho_{1} \alpha_{1}^{2}-2 \rho_{1} \mu_{1}+C \rho_{1}^{2} \alpha_{1}^{2}}+\frac{\tau_{2}}{r_{2} t_{2}} \rho_{2} \xi_{2} \gamma_{1},\right. \\
& \left.\frac{\tau_{2}}{r_{2} t_{2}} \sqrt{\beta_{2}^{2}+2 c_{2} \rho_{2} \alpha_{2}^{2}-2 \rho_{2} \mu_{2}+C \rho_{2}^{2} \alpha_{2}^{2}}+\frac{\tau_{1}}{r_{1} t_{1}} \rho_{1} \xi_{1} \gamma_{2}\right\} .
\end{aligned}
$$


The assumption (4.21) assures that $0<\theta<1$. Therefore, by (4.30) and $0<\lambda_{k}+\delta_{k} \leq 1$ implies that $\left\{a_{k}\right\}$ and $\left\{b_{k}\right\}$ are both Cauchy sequences. Since the spaces $X_{1}$ and $X_{2}$ are complete, there exist $a \in X_{1}$ and $b \in X_{2}$ such that $a_{k} \rightarrow a$ and $b_{k} \rightarrow b$ as $k \rightarrow \infty$.

Next we prove that $u_{k} \rightarrow u \in U(a)$ and $v_{k} \rightarrow v \in V(b)$ as $k \rightarrow \infty$. The inequalities (4.26) and (4.27) guarantee that $\left\{u_{k}\right\}$ and $\left\{v_{k}\right\}$ are also Cauchy sequences. Therefore there exist $u \in X_{1}$ and $v \in X_{2}$ such that $u_{k} \rightarrow u$ and $v_{k} \rightarrow v$ as $k \rightarrow \infty$. Moreover,

$$
\begin{aligned}
d(u, U(a))=\inf \{\|u-t\|: t \in U(a)\} & \leq\left\|u-u_{k}\right\|+d\left(u_{k}, U(a)\right) \\
& \leq\left\|u-u_{k}\right\|+\mathcal{D}_{1}\left(U\left(a_{k}\right), U(a)\right) \\
& \leq\left\|u-u_{k}\right\|+\xi_{1}\left\|a_{k}-a\right\| \rightarrow 0 .
\end{aligned}
$$

Since $U(a)$ is closed, we have $u \in U(a)$. Similarly, we can show that $v \in V(b)$.

Finally, applying the continuity property of $A_{1}, F, A_{2}, G$ and the continuity of the generalized resolvent operator, we get

$$
\left\{\begin{array}{l}
a=J_{\rho_{1}, M_{1}}^{A_{1}, \eta_{1}}\left(A_{1}(a)-\rho_{1} F(a, v)\right), \\
b=J_{\rho_{2}, M_{2}}^{A_{2}, \eta_{2}}\left(A_{2}(a)-\rho_{2} G(u, b)\right) .
\end{array}\right.
$$

Because of Theorem 4.1, we conclude that $(a, b, u, v)$ is a solution of the variational inclusion problem (3.3).

If we replace the relaxed cocoercivity condition by strong monotonicity condition on the maps $F(., b)$ and $G(x,$.$) , then we get the following result. We write it in the form of a corollary.$

\section{Corollary 4.2}

Let $\eta_{i}: X_{i} \times X_{i} \rightarrow X_{i}$ be $t_{i}$-strongly monotone and $\tau_{i}$-Lipschitz continuous, $A_{i}: X_{i} \rightarrow X_{i}$ be $r_{i}$-strongly monotone and $\beta_{i}$-Lipschitz continuous, and let $M_{i}: X_{i} \rightarrow 2^{X_{i}}$ be relative $\left(A_{i}, \eta_{i}\right)$-maximal monotone mappings for $i=1,2$. Let $U: X_{1} \rightarrow C\left(X_{1}\right)$ be $\mathcal{D}_{1}-\gamma_{1}$-Lipschitz continuous and $V: X_{2} \rightarrow C\left(X_{2}\right)$ be $\mathcal{D}_{2}-\gamma_{2}$-Lipschitz continuous. Let $F: X_{1} \times X_{2} \rightarrow X_{1}$ be a nonlinear mapping such that for any given $(a, b) \in X_{1} \times X_{2}, F(., b)$ is $\mu_{1}$-strongly monotone with respect to $A_{1}$ and $\alpha_{1}$-Lipschitz continuous, and $F(a,$.$) is \xi_{1}$-Lipschitz continuous. Let $G: X_{1} \times X_{2} \rightarrow X_{2}$ be another nonlinear mapping such that for any given $(x, y) \in X_{1} \times X_{2}, G(x,$.$) is \mu_{2}$-strongly monotone with respect to $A_{2}$ and $\alpha_{2}$-Lipschitz continuous, and $G(., y)$ is $\xi_{2}$-Lipschitz continuous. If there exist positive real constants $\rho_{1}, \rho_{2}$ such that

$$
\left\{\begin{array}{l}
\tau_{1} r_{2} t_{2} \sqrt{\beta_{1}^{2}-2 \rho_{1} \mu_{1}+C \rho_{1}^{2} \alpha_{1}^{2}}+\tau_{2} r_{1} t_{1} \rho_{2} \xi_{2} \gamma_{1}<r_{1} r_{2} t_{1} t_{2}, \\
\tau_{2} r_{1} t_{1} \sqrt{\beta_{2}^{2}-2 \rho_{2} \mu_{2}+C \rho_{2}^{2} \alpha_{2}^{2}}+\tau_{1} r_{2} t_{2} \rho_{1} \xi_{1} \gamma_{2}<r_{1} r_{2} t_{1} t_{2} .
\end{array}\right.
$$

Then the variational inclusion problem (3.3) has a solution $(a, b, u, v)$. The iterative sequences $\left\{a_{k}\right\},\left\{b_{k}\right\},\left\{u_{k}\right\},\left\{v_{k}\right\}$ generated by Algorithm 4.1 converge strongly to the solution $(a, b, u, v)$.

Next we study the solvability of the system of variational inclusion problems (3.3) involving $(H, \eta)$-maximal monotonicity instead of $(A, \eta)$-maximal relaxed monotonicity. As in the earlier case, we convert the problem to another equivalent form. Then construct an iterative algorithm using the equivalent form. Finally, we study the convergency of the iterative method to the solution of the problem (3.3). The idea of the proof are similar. So we state the results without proof.

Let $X_{1}$ and $X_{2}$ are 2-uniformly smooth Banach spaces. Let $F: X_{1} \times X_{2} \rightarrow X_{1}, G: X_{1} \times X_{2} \rightarrow X_{2}, H_{i}: X_{i} \rightarrow$ $X_{i}$ and $\eta_{i}: X_{i} \times X_{i} \rightarrow X_{i}(i=1,2)$ be nonlinear single valued mappings. Let $U: X_{1} \rightarrow 2^{X_{1}}, V: X_{2} \rightarrow 2^{X_{2}}$ be set valued mappings, and let $M_{i}: X_{i} \rightarrow 2^{X_{i}}$ be $\left(H_{i}, \eta_{i}\right)$-maximal monotone mappings $(i=1,2)$.

Theorem 4.4

Let $(a, b) \in X_{1} \times X_{2}, u \in U(a), v \in V(b)$. Then $(a, b, u, v)$ is a solution of the problem (3.3) if and only if $(a, b, u, v)$ satisfies

$$
\left\{\begin{array}{l}
a=J_{\rho_{1}, M_{1}}^{H_{1}, \eta_{1}}\left(H_{1}(a)-\rho_{1} F(a, v)\right), \\
b=J_{\rho_{2}, M_{2}}^{H_{2}, \eta_{2}}\left(H_{2}(a)-\rho_{2} G(u, b)\right),
\end{array}\right.
$$

Stat., Optim. Inf. Comput. Vol. 5, September 2017 
where $\rho_{1}$ and $\rho_{2}$ are two positive constants.

Using the above theorem, we can able to construct the following algorithm:

\section{Algorithm 4.2}

Step 1: Choose initial approximation $\left(a_{0}, b_{0}\right) \in X_{1} \times X_{2}$ and $u_{0} \in U\left(a_{0}\right), v_{0} \in V\left(b_{0}\right)$.

Step 2: Construct the iteration

$$
\left\{\begin{array}{l}
a_{k+1}=\left(1-\lambda_{k}-\delta_{k}\right) a_{k}+\lambda_{k} J_{\rho_{1}, M_{1}}^{H_{1}, \eta_{1}}\left(H_{1}\left(a_{k}\right)-\rho_{1} F\left(a_{k}, v_{k}\right)\right), \\
b_{k+1}=\left(1-\lambda_{k}-\delta_{k}\right) b_{k}+\lambda_{k} J_{\rho_{2}, M_{2}}^{H_{2}, \eta_{2}}\left(H_{2}\left(b_{k}\right)-\rho_{2} G\left(u_{k}, b_{k}\right)\right),
\end{array}\right.
$$

where $\lambda_{k}$ and $\delta_{k}$ are positive real constant such that $0<\lambda_{k}+\delta_{k} \leq 1$, and

$\lim \sup _{k \geq 0} \lambda_{k}<1$.

Step 3: Choose $u_{k+1} \in U\left(a_{k+1}\right)$ and $v_{k+1} \in V\left(b_{k+1}\right)$ such that

$$
\left\{\begin{array}{l}
\left\|u_{k+1}-u_{k}\right\| \leq\left(1+(1+k)^{-1}\right) \mathcal{D}_{1}\left(U\left(a_{k+1}\right), U\left(a_{k}\right)\right), \\
\left\|v_{k+1}-v_{k}\right\| \leq\left(1+(1+k)^{-1}\right) \mathcal{D}_{2}\left(V\left(b_{k+1}\right), V\left(b_{k}\right)\right),
\end{array}\right.
$$

where $\mathcal{D}_{i}(.,$.$) is the Hausdorff pseudo-metric on 2^{X_{i}}$ for $i=1,2$.

Step 4: If $a_{k+1}, b_{k+1}, u_{k+1}, v_{k+1}$ satisfy (4.32) to a sufficient degree of accuracy, then stop; else set $k=k+1$ and return to Step 2.

\section{Theorem 4.5}

Let $\eta_{i}: X_{i} \times X_{i} \rightarrow X_{i}$ be $\tau_{i}$-Lipschitz continuous, $H_{i}: X_{i} \rightarrow X_{i}$ be $\left(r_{i}, \eta_{i}\right)$-strongly monotone and $\beta_{i}$-Lipschitz continuous, and let $M_{i}: X_{i} \rightarrow 2^{X_{i}}$ be $\left(H_{i}, \eta_{i}\right)$-maximal monotone mappings for $i=1,2$. Let $U: X_{1} \rightarrow C\left(X_{1}\right)$ be $\mathcal{D}_{1}-\gamma_{1}$-Lipschitz continuous and $V: X_{2} \rightarrow C\left(X_{2}\right)$ be $\mathcal{D}_{2}-\gamma_{2}$-Lipschitz continuous. Let $F: X_{1} \times X_{2} \rightarrow X_{1}$ be a nonlinear mapping such that for any given $(a, b) \in X_{1} \times X_{2}, F(., b)$ is $\left(c_{1}, \mu_{1}\right)$-relaxed cocoercive with respect to $H_{1}$ and $\alpha_{1}$-Lipschitz continuous, and $F(a,$.$) is \xi_{1}$-Lipschitz continuous. Let $G: X_{1} \times X_{2} \rightarrow X_{2}$ be another nonlinear mapping such that for any given $(x, y) \in X_{1} \times X_{2}, G(x,$.$) is \left(c_{2}, \mu_{2}\right)$-relaxed cocoercive with respect to $H_{2}$ and $\alpha_{2}$-Lipschitz continuous, and $G(., y)$ is $\xi_{2}$-Lipschitz continuous. If there exist positive real constants $\rho_{1}, \rho_{2}$ such that

$$
\left\{\begin{array}{l}
\tau_{1} r_{2} \sqrt{\beta_{1}^{2}+2 c_{1} \rho_{1} \alpha_{1}^{2}-2 \rho_{1} \mu_{1}+C \rho_{1}^{2} \alpha_{1}^{2}}+\tau_{2} r_{1} \rho_{2} \xi_{2} \gamma_{1}<r_{1} r_{2}, \\
\tau_{2} r_{1} \sqrt{\beta_{2}^{2}+2 c_{2} \rho_{2} \alpha_{2}^{2}-2 \rho_{2} \mu_{2}+C \rho_{2}^{2} \alpha_{2}^{2}}+\tau_{1} r_{2} \rho_{1} \xi_{1} \gamma_{2}<r_{1} r_{2} .
\end{array}\right.
$$

Then the variational inclusion problem (3.3) has a solution $(a, b, u, v)$. The iterative sequences $\left\{a_{k}\right\},\left\{b_{k}\right\},\left\{u_{k}\right\},\left\{v_{k}\right\}$ generated by Algorithm 4.2 converge strongly to the solution $(a, b, u, v)$.

\section{Conclusion}

A new system of nonlinear variational inclusion problems involving $(A, \eta)$-maximal relaxed monotone mappings has been introduced in 2-uniformly smooth Banach spaces. Using the generalized resolvent operator technique, an iterative algorithm has been constructed to solve the proposed system, and the convergence analysis of the algorithm has been investigated. Moreover the obtained results are generalized to solve the system of variational inclusions involving relative $(A, \eta)$-maximal relaxed monotone mappings. The obtained results generalize most of the results investigated in the literature, and offer a wide range of applications to future research on the sensitivity analysis, variational inclusion problems, variational inequality problems in Banach spaces.

\section{Acknowledgement}

The authors are thankful to the referees for their valuable suggestions that improved the presentation of the paper. 


\section{REFERENCES}

1. S. Adly, Perturbed algorithm and sensitivity analysis for a general class of variational inclusions, J. Math. Anal. Appl., vol. 201, pp. 609-630, 1996.

2. R. P. Agarwal, and R. U. Verma, General system of $(A, \eta)$-maximal relaxed monotone variational inclusion problems based on generalized hybrid algorithms, Commun. Nonlinear Sci. Numer. Simulat., vol. 15, pp. 238-251, 2010.

3. I. Ahmad, M. Rahaman, and R. Ahmad, Relaxed resolvent operator for solving variational inclusion problem, Stat. Optim. Inf. Comput., vol. 4, pp. 183-193, 2016.

4. Y. J. Cho, Y. P. Fang, N. J. Huang, and H. J. Hwang, Algorithms for systems of nonlinear variational inequalities, J. Korean Math. Soc., vol. 41, pp. 489-499, 2004.

5. X. P. Ding, Perturbed proximal point for generalized quasi-variational inclusions, J. Math. Anal. Appl., vol. 210, pp. 88-101, 1997.

6. X. P. Ding, and C. L. Luo, Perturbed proximal point algorithms for generalized quasi-variational like inclusions, J. Comput. Appl. Math., vol. 210, pp. 153-165, 2000.

7. Y. P. Fang, and N. J. Huang, H-monotone operator and resolvent operator technique for variational inclusions, Appl. Math. Comput., vol. 145, pp. 795-803, 2003.

8. J. R. Giles, Classes of semi-inner product spaces, Trans. Amer. Math. Soc., vol. 129, pp. 436-446, 1967.

9. N. J. Huang, A new class of generalized set valued implicit variational inclusions in Banach spaces with an application, Comput. Math. Appl., vol. 41, pp. 937-943, 2001.

10. D. O. Koehler, A note on some operator theory in certain semi-inner product spaces, Proc. Amer. Math. Soc., vol. 30, pp. 363-366, 1971.

11. H. Y. Lan, J. H. Kim, and Y. J. Cho, On a new system of nonlinear A-monotone multivalued variational inclusions, J. Math. Anal. Appl., vol. 327, pp. 481-493, 2007.

12. C. H. Lee, Q. H. Ansari, and J. C. Yao, A perturbed algorithm for strongly nonlinear variational like inclusions, Bull. Aust. Math. Soc., vol.62, pp. 417-426, 2000.

13. G. Lumer, Semi-inner product spaces, Trans. Amer. Math. Soc., vol. 100, pp. 29-43, 1961.

14. J. Peng, and D. Zhu, A new system of generalized mixed quasi-variational inclusions with (H, $\eta)$-monotone operators, J. Math. Anal. Appl., vol. 327, pp. 175-187, 2007.

15. N. K. Sahu, R. N. Mohapatra, C. Nahak, and S. Nanda, Approximation solvability of a class of A-monotone implicit variational inclusion problems in semi-inner product spaces, Appl. Math. Comput., vol. 236, pp. 109-117, 2014.

16. R. U. Verma, Approximation solvability of a class of nonlinear set-valued variational inclusions involving $(A, \eta)$-monotone mappings, J. Math. Anal. Appl., vol. 337, pp. 969-975, 2008.

17. R. U. Verma, Sensitivity analysis for generalized strongly monotone variational inclusions based on $(A, \eta)$-resolvent operator technique, Appl. Math. Lett., vol. 19, pp. 1409-1413, 2006.

18. H. K. Xu, Inequalities in Banach spaces with applications, Nonlinear Analysis: Theory, Methods and Applications, vol. 16, pp. 1127-1138, 1991. 